Original research article

\title{
RIG-1 and MDA5 are the important intracellular sensors against bacteria in septicemia suffering patients
}

\author{
Asieh Asadpour-Behzadi, Ashraf Kariminik* \\ Department of Microbiology,Kerman Branch, Islamic Azad University, Kerman, Iran
}

\section{A R T I C L E I N F O}

\section{Article history:}

Received 4 September 2017

Received in revised form 29 November 2017

Accepted 31 January 2018

Available online 21 February 2018

\section{Keywords:}

Septicemia

MDA5

RIG-1

Innate immunity

Inflammation

\begin{abstract}
A B S T R A C T
Background: MDA5 and RIG-1 are the important intracellular receptors which detect microbial associated molecular patterns. Septicemia is a condition in which infection enters the bloodstream of the patients. The main intracellular mechanisms against septicemia are yet to be clarified. Therefore, this research study was aimed to evaluate expression of MDA5 and RIG-1 in the patients suffering from septicemia in comparison to healthy controls.

Methods: MDA5 and RIG-1 expression levels in 40 patients suffering from septicemia and 40 healthy controls were evaluated using Real-Time PCR technique. The sources of bacteria in the bloodstream of the patients suffering from septicemia were determined using microbial cultures.

Results: The results showed that mRNA levels of MDA5 and RIG-1 were significantly increased in the patients when compared to healthy controls. The results also revealed that the patients were infected with four bacteria including Escherichia coli,Staphylococcus aureus, Acinetobacter baumannii and Pseudomonas aeruginosa. mRNA levels of MDA5 and RIG-1 did not differ among patients with various bacterial infections.

Conclusion: Based on the results it seems that MDA5 and RIG-1 are the main intracellular immunity against the bacteria during septicemia and could be considered for their roles in induction of immunity. (c) 2018 Faculty of Health and Social Sciences, University of South Bohemia in Ceske Budejovice. Published by Elsevier Sp. z o.o. All rights reserved.
\end{abstract}

\section{Introduction}

It has been demonstrated that intracellular sensors, as main pattern recognition receptors (PRRs), participate in recognition of pathogens, and hence, can induce appropriate immune responses to eradicate infection (Bagheri et al., 2014; Karimi-Googheri and Arababadi, 2014). Recent investigations revealed that melanoma differentiation-associated protein 5 (MDA5) and retinoic acidinducible gene 1 (RIG-1) are expressed in the cytoplasm of immune cells and play key roles in recognition of pathogen associated molecular patterns (PAMPs) (Ebrahim et al., 2015). Interferon regulatory transcription factor 3 (IRF3) and nuclear factor-kappaB $(\mathrm{NF}-\mathrm{kB})$ are the main down-stream pro-inflammatory transcription factors of MDA5 and RIG-1 (Bustos-Arriaga et al., 2011). Thus, they may play key roles in induction of immune responses against bacteria in human. It has been determined that innate immune responses are the main criteria for eradication of infections from tissues, including blood (septicemia) (Chantratita et al., 2017; Lewis et al., 2016). Accordingly, it is worthy to clarify the main

\footnotetext{
* Author for correspondence: Islamic Azad University, Kerman Branch, Department of Microbiology, Kerman, Iran.

E-mail address: a.kariminik@iauk.ac.ir (A. Kariminik).
}

immune responses elements against septicemia to determine the main parameters of immune responses which are involved during the disorder. Based on the important roles played by MDA5 and RIG-1 as intracellular receptors to activate innate immune responses (Dou et al., 2017; Loo et al., 2008), it seems that the molecules may participate in the induction of immune responses against bacteria during septicemia. Therefore, the aim of this study was to determine the expression levels of MDA5 and RIG- 1 in patients suffering from septicemia in comparison to healthy controls.

\section{Materials and methods}

\section{Subjects}

This cross-sectional study was performed on 40 patients suffering from septicemia and 40 sex and age matched healthy controls. Septicemia was approved by an expert MD in infectious diseases using the following criteria: Positive bacteria blood culture, existence of fever, respiratory distress, hypothermia, jaundice, cyanosis, lethargy, apnoea, convulsion, irritability, vomiting and poor feeding (Farhat et al., 2014). Positive bacteria blood culture and fever were seen in all the patients, while other 
criteria were seen in some patients. The samples were collected from patients with septicemia before antibiotic therapy which were selected from Afzalipour hospital of Kerman city, Kerman, Iran. The patients who were pregnant, breast feeding, under administration of immune affected drugs, cancers, allergies, diabetes and autoimmunity were excluded from the study. 12 and $2 \mathrm{ml}$ of blood were taken from the patients and controls, respectively. The blood from the patients was used for blood culture $(10 \mathrm{ml})$ and mRNA extraction $(2 \mathrm{ml})$, while all of the blood samples from controls were used for mRNA extraction only. The Ethical Committee of Islamic Azad University, Kerman Branch approved the protocol study and all patients with septicemia and those in control group filled out the concerned written informed consent form.

\section{Blood culture}

For blood culture, $10 \mathrm{ml}$ of blood was taken from the suspected patients and injected into two culture media, Thioglycollate broth (Merck, Germany) simultaneously, and were incubated in either aerobic or anaerobic conditions at $37^{\circ} \mathrm{C}$ for 7 days. The bottles were observed daily regarding microbial growth and its evidence including gas production, hemolysis, media turbidity, formation of discrete colonies, etc. Additionally, the samples were also subcultured and incubated onto MacConkey agar and Blood agar at $37^{\circ} \mathrm{C}$ for $24-48 \mathrm{~h}$ to identify the pathogenic bacteria. The positive cultures were selected for diagnostic bacteriological tests (Tille, 2013). All the media was prepared from Merck Company, Berlin, Germany.

\section{Real-time PCR}

The collected samples (whole blood) in anti-coagulant (Ethylenediaminetetraacetic acid (EDTA)) pre-treated tubes were used for RNA purification by a commercial kit from Cinnaclon Company, Tehran, Iran. The extracted RNA was converted to cDNA using a commercial kit (Parstoos Com, Tehran, Iran). Real-time PCR was performed based on our previous investigation protocol (Kariminik et al., 2016), except the used housekeeping gene, $\beta$-actin. The primer sequences which were used were as follow: RIG-1 Forward: CACACCAAGAGCCCAAAC, RIG-1 Reverse: TGACCCGATAGCAACAGC, MDA5 Forward: GCAGAGGTGAAGGAGCAGA, MDA5 Reverse: AAACGATGGAGAGGGCAAG, $\beta$-actin Forward: GGCACCCAGCACAATGAAG, $\beta$-actin Reverse: CCGATCCACACGGAGTACTTG. In other words, $\beta$-actin was used as internal control. Briefly, in order to extract mRNA, $200 \mu \mathrm{l}$ of whole blood was lysed by lysis buffer and transmitted from RNA purification filters by centrifuge at $12000 \mathrm{~g}$ for $8 \mathrm{~min}$. Filters were washed by appropriate washing buffer which was prepared in the kits and finally, the purified mRNA was separated from the filters using elution buffer. The purified mRNA was used for cDNA synthesize using oligo-dT primers and a commercial kit from Parstoos Com, Tehran, Iran. Accordingly, the purified mRNA, oligo-dT primer and pre-mix solution (containing dNTPs and reverse transcriptase) was incubated at $40^{\circ} \mathrm{C}$ for $60 \mathrm{~min}$. The synthesized cDNA has been used for amplification in a Real-Time PCR instrument (Rotor-Gene $\mathrm{Q}$ series) using the mentioned primers and a mastermix from Parstoos Com, Tehran, Iran using the following program: $5 \mathrm{~min}$ at $96^{\circ} \mathrm{C}$ (one cycle), 30,30 and $40 \mathrm{~s}$ at $96^{\circ} \mathrm{C}, 58^{\circ} \mathrm{C}$ and $72^{\circ} \mathrm{C}$ respectively, for 40 cycles. The raw data were analysed using $2^{-\Delta \Delta c t}$ formula.

\section{Data analysis and statistical methods}

The differences between the patients with septicemia and healthy controls and female in comparison to male were calculated using Mann-Whitney $U$ test, and the differences among the patients infected with various bacteria were examined using Kruskal-Wallis test under SPSS software version 18. P-value was considered significant at $<0.05$. Data are presented in the result section as median (1st quartile-3rd quartile).

\section{Results}

Identification of septicaemia

Based on blood culture and diagnostic tests, the following bacteria were identified: Escherichia coli (7/40 cases), Staphylococcus aureus (10/40 cases), Acinetobacter baumannii (17/40 cases) and Pseudomonas aeruginosa (6/40 cases).

\section{Expression levels of target genes}

Results showed that MDA5 mRNA levels in the peripheral blood immune cells (PBIC) of the patients suffering from septicemia and healthy controls were 7.4108 (1.0611-184.4549) and 0.1959 (0.0263-0.6239), respectively. Statistical analysis demonstrated a significant difference $(p<0.001)$. Expression levels of RIG-1 also significantly $(p<0.001)$ increased in the septicemia patients (7.4054 (0.1190-579.4633)) when compared to healthy controls (0.0040 (0.0007-0.0339)). Fig. 1 illustrates the expression levels of MDA5 and RIG-1 in the septicemia patients and healthy controls.
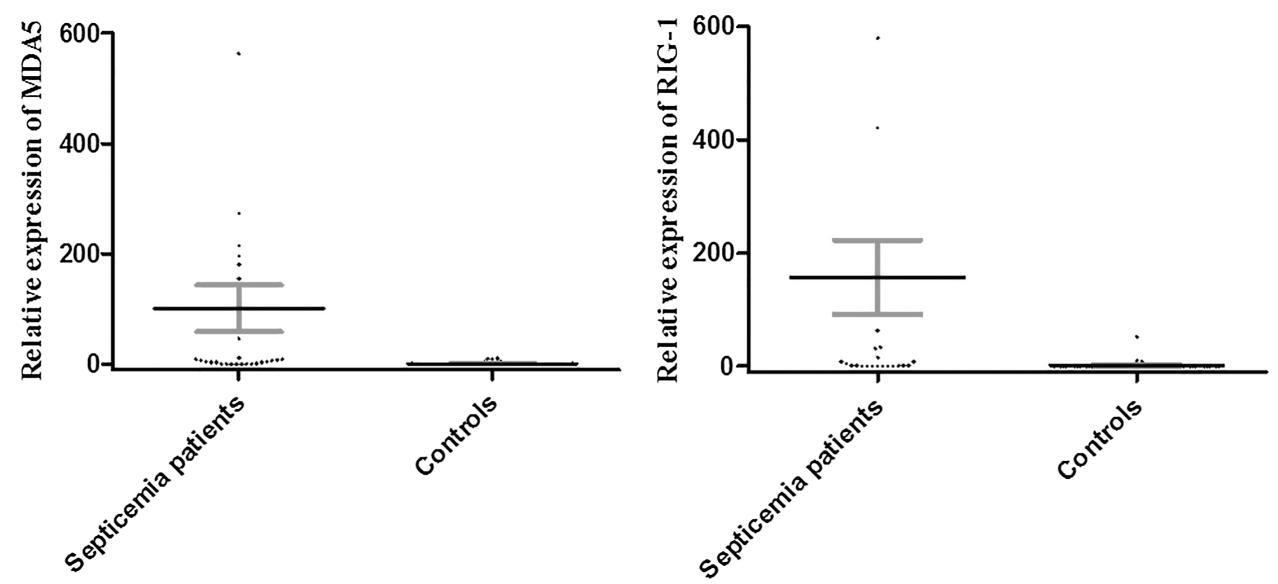

Fig. 1. Expression levels of MDA5 and RIG-1 in septicemia patients and healthy controls. Figure illustrates that the mRNA levels of MDA5 and RIG-1 were significantly increased in septicemia patients in comparison to healthy controls. 

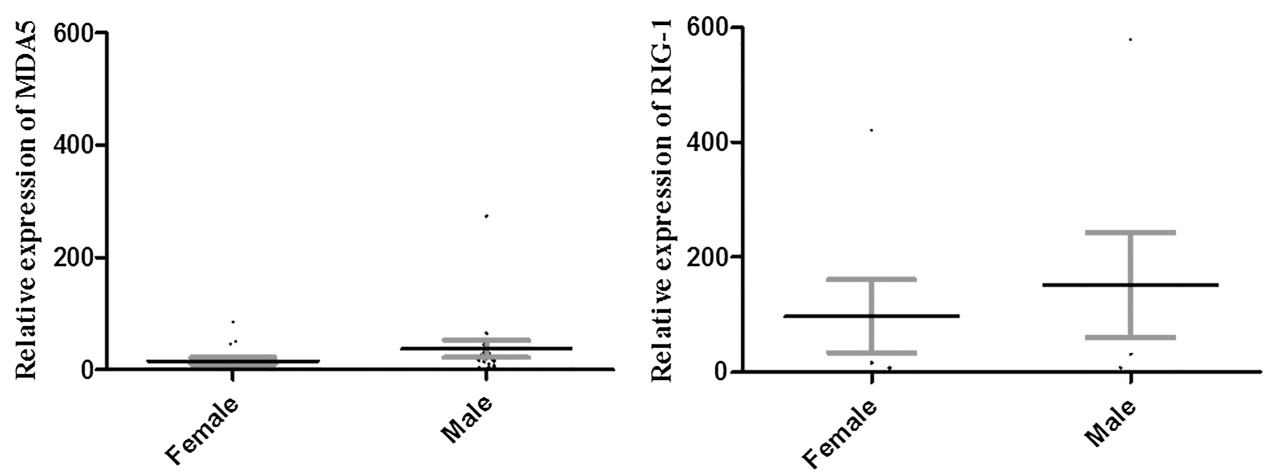

Fig. 2. Expression levels of MDA5 and RIG-1 in female in comparison to male septicemia patients. Figure illustrates that the mRNA levels of MDA5 and RIG-1 were not significantly different in female and male septicemia patients.
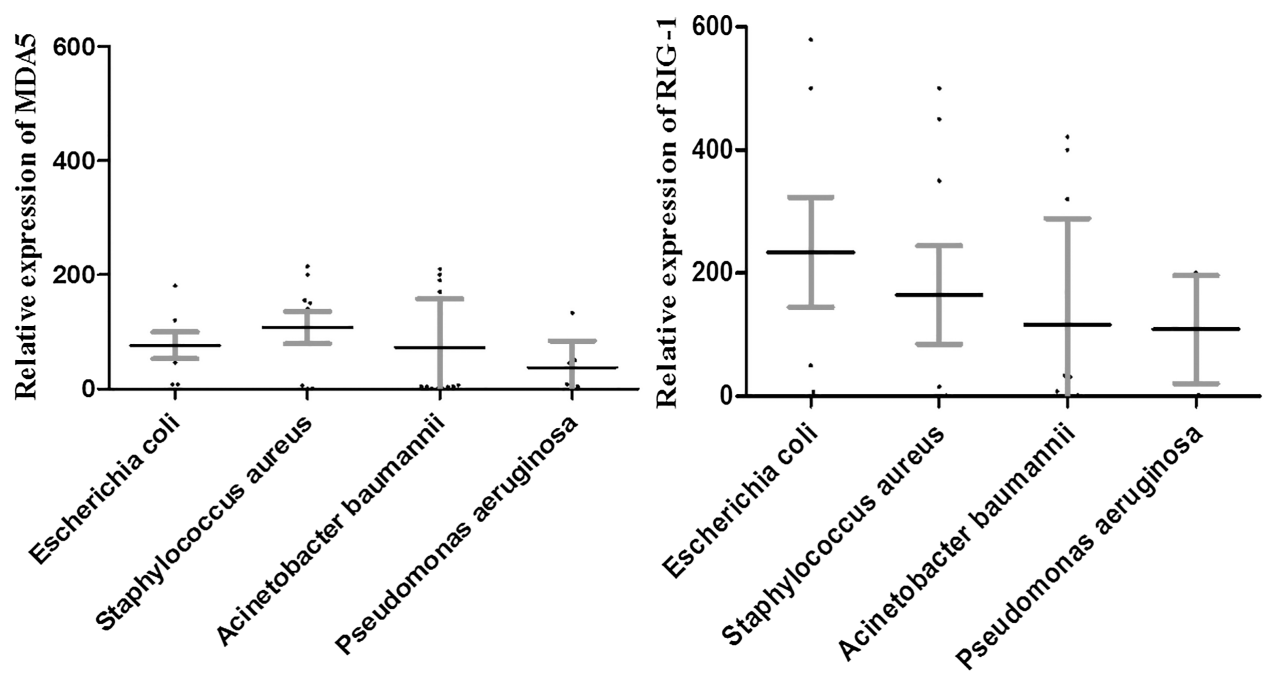

Fig. 3. Expression levels of MDA5 and RIG-1 in septicemia patients infected with various Escherichia coli, Staphylococcus aureus, Acinetobacter baumannii and Pseudomonas aeruginosa. The figure illustrates that the mRNA levels of MDA5 and RIG-1 did not significantly differ among groups.

The results revealed that mRNA levels of MDA5 $(p=0.018)$ were significantly higher in the female (4.7442 (0.4685-8.4924)) when compared to male (180.5401 (3.5955-562.6819)) septicemia patients (Fig. 2).

Expression levels of RIG-1 $(p=0.236)$ did not significantly differ between female $(0.0027(0.0863-0.0007))$ when compared to male (0.0048 (0.0006-0.0103)) septicemia patients (Fig. 2).

Results also demonstrated that expression levels of MDA5 ( $p=0.303)$ and RIG-1 $(p=0.576)$ did not significantly differ among septicemia patients infected with various bacteria (Escherichia coli, Staphylococcus aureus, Acinetobacter baumannii and Pseudomonas aeruginosa). Fig. 3 illustrates the expression levels of MDA5 and RIG-1 in the septicemia patients infected with various pathogens.

The results also revealed that there was no significant correlation between age and expression of MDA5 and RIG- 1 in the septicemia patients (Table 1 ).

The results also showed that male and female septicemia patients were similar regarding the type of infected bacteria (Table 2).

\section{Discussion}

Septicemia is a condition with distributed infection in the bloodstream (Fecteau et al., 2009; Sogaard et al., 2012). The results showed that the septicemia patients have a significant increased expression of both MDA5 and RIG-1. As mentioned previously, MDA5 and RIG-1 are the important intracellular receptors which play key roles in recognition of microbes (Hu et al., 2017) and, hence, can be considered as important factors against septicemia. Results of the current study proved the hypothesis and revealed that mRNA levels of the intracellular sensors have been increased following septicemia. Accordingly, it seems that either MDA5 or RIG-1 participate in induction of immune responses against the bacteria

Table 1

Correlation between age and mRNA levels of MDA5 and RIG-1 in septicemia patients.

\begin{tabular}{llll}
\hline & & Male & Female \\
\hline \multirow{2}{*}{ Bacteria } & Escherichia coli & 3 & 4 \\
& Staphylococcus aureus & 4 & 5 \\
& Acinetobacter baumannii & 9 & 9 \\
& Pseudomonas aeruginosa & 2 & 4 \\
\hline
\end{tabular}

Table illustrates that there was no significant correlation between age and mRNA levels of MDA5 and RIG-1 in septicemia patients.

Table 2

Distribution of bacterial infection between male and female participants.

\begin{tabular}{lllll}
\hline & & MDA5 & RIG-1 \\
\hline \multirow{2}{*}{ Spearman's rho } & \multirow{2}{*}{ Age } & Correlation coefficient & 0.034 & -0.030 \\
& & $P$ value & 0.871 & 0.889 \\
\hline
\end{tabular}

The table demonstrates that male and female were no different regarding the pattern of bacterial infection. 
which is responsible for septicemia. Based on the fact that both molecules recognize microbial RNAs, it seems that one of the main immune responses against bacteria is related to recognition of bacterial RNA during septicemia. To the best of our knowledge, this is the first project which evaluated the roles of MDA5 and RIG-1 in the immune responses against septicemia but some investigations revealed that down-stream molecules and target genes of MDA5 and RIG-1 are involved in the responses during septicemia. For instance, studies reported that septicemia patients infected with Escherichia coli up-regulate expression of pro-inflammatory cytokines in a Nuclear factor- $\kappa \mathrm{B}(\mathrm{NF}-\kappa \mathrm{B})$ dependent manner (Gao et al., 2016; Selvaraj et al., 2015). NF-кB is a transcription factor which is activated by several pathways including MDA5 and RIG-1 dependent pathways (Matsukura et al., 2007). Additionally, the roles played by MDA5 and RIG-1 against other pathogens rather than bacteria including parasites (Jaeger et al., 2015; Wu et al., 2014) and viruses (Ebrahim et al., 2015; Ohtani et al., 2011) have been reported previously. Therefore, the roles of MDA5 and RIG-1 in the fight against bacteria in the septicemia are plausible and further investigations could illustrate a better understanding of the mechanisms used by the molecules during septicemia. The results also revealed that expression levels of MDA5 but not RIG-1 were significantly increased in female in comparison to male patients. Accordingly, it may be concluded that sex is an important factor to interfere with septicemia in a MDA5 dependent manner. Additionally, results proved that the expression of both MDA5 and RIG-1 molecules in the septicemia patients infected with various bacteria did not differ; hence, it seems that the kind of the bacteria cannot be considered as a critical factor for expression of the evaluated molecules during septicemia. Moreover, data analysis demonstrated that age also has no effects on the expression of the MDA5, which is a sex dependent factor, as well as RIG-1.

However, based on the fact that MDA5 and RIG-1 use some intracellular signalling molecules as down-stream molecules such as receptor interacting protein 1 (RIP1) and IFN- $\beta$ promoter stimulator1 (IPS-1) (Swiecki et al., 2011), it is worthy to evaluate the molecules and their related transcription factors, interferon regulatory factor (IRF) 3 and 5, in the patients with septicemia. Additionally, it may be hypothesized that molecular therapy which targets MDA5 and RIG-1 and their related molecules might be considered as a new strategy to decrease the complication of septicemia.

\section{Conflict of interests}

The authors have no conflict of interests to declare.

\section{Acknowledgements}

This project was supported by a grant from the Islamic Azad University, Kerman Branch, Kerman, Iran.

\section{References}

Bagheri, V., Askari, A., Arababadi, M.K., Kennedy, D., 2014. Can toll-like receptor (TLR) 2 be considered as a new target for immunotherapy against hepatitis $B$ infection? Hum. Immun. 75, 549-554.

Bustos-Arriaga, J., Garcia-Machorro, J., Leon-Juarez, M., Garcia-Cordero, J., SantosArgumedo, L., Flores-Romo, L., et al., 2011. Activation of the innate immune response against DENV in normal non-transformed human fibroblasts. PLoS Negl.Trop. Dis. 5, e1420.

Chantratita, N., Tandhavanant, S., Seal, S., Wikraiphat, C., Wongsuvan, G., Ariyaprasert, P., et al., 2017. TLR4 genetic variation is associated with inflammatory responses in gram-positive sepsis. Clin. Microbiol. Infect. 23 (1) 47. e1-47. e10.

Dou, Y., Yim, H.C., Kirkwood, C.D., Williams, B.R., Sadler, A.J., 2017. The innate immune receptor MDA5 limits rotavirus infection but promotes cell death and pancreatic inflammation. EMBO J. 36, 2742-2757.

Ebrahim, M., Mirzaei, V., Bidaki, R., Shabani, Z., Daneshvar, H., Karimi-Googheri, M., et al., 2015. Are RIG-1 and MDA5 expressions associated with chronic HBV infection? Viral Immunol. 28, 504-508.

Farhat, A.S., Mohammadzadeh, A., Mirzaie, F., Khademi, G., Nasab, M.N., 2014. Clinical manifestation and laboratory findings of positive blood culture in neonatal septicemia. Iran. J. Neonatol. 5, 14.

Fecteau, G., Smith, B.P., George, L.W., 2009. Septicemia and meningitis in the newborn calf. Vet. Clin. North Am. Food Anim. Pract. 25, 195-208.

Gao, F., Yang, Y.-Z., Feng, X.-Y., Fan, T.-T., Jiang, L., Guo, R., et al., 2016. Interleukin-27 is elevated in sepsis-induced myocardial dysfunction and mediates inflammation. Cytokine 88, 1-11.

Hu, M.M., Liao, C.Y., Yang, Q., Xie, X.Q., Shu, H.B., 2017. Innate immunity to RNA virus is regulated by temporal and reversible simulation of RIG-I and MDA5. J. Exp. Med. 214, 973-989.

Jaeger, M., van der Lee, R., Cheng, S.C., Johnson, M.D., Kumar, V., Ng, A., et al., 2015 The RIG-I-like helicase receptor MDA5 (IFIH1) is involved in the host defence against Candida infections. Eur. J. Clin. Microbiol. 34, 963-974.

Karimi-Googheri, M., Arababadi, M.K., 2014. TLR3 plays significant roles against hepatitis B virus. Mol. Biol. Rep. 41, 3279-3286.

Kariminik, A., Dabiri, S., Yaghobi, R., 2016. Polyomavirus BK induces inflammation via up-regulation of CXCL10 at translation levels in renal transplant patients with nephropathy. Inflammation 39, 1514-1519.

Lewis, A.J., Billiar, T.R., Rosengart, M.R., 2016. Biology and metabolism of sepsis: innate immunity bioenergetics, and autophagy. Surg. Infect. 17, 286-293.

Loo, Y.M., Fornek, J., Crochet, N., Bajwa, G., Perwitasari, O., Martinez-Sobrido, L., et al., 2008. Distinct RIG-I and MDA5 signalling by RNA viruses in innate immunity. J. Virol. 82, 335-345.

Matsukura, S., Kokubu, F., Kurokawa, M., Kawaguchi, M., Ieki, K., Kuga, H., et al., 2007. Role of RIG-I, MDA-5, and PKR on the expression of inflammatory chemokines induced by synthetic dsRNA in airway epithelial cells. Int. Arch. Allergy Immunol. 143 (Suppl. 1), 80-83.

Ohtani, M., Hikima, J., Kondo, H., Hirono, I., Jung, T.S., Aoki, T., 2011. Characterization and antiviral function of a cytosolic sensor gene MDA5, Japanese flounder, Paralichthys olivaceus. Dev. Comp. Immunol. 35, 554-562.

Selvaraj, V., Manne, N.D., Arvapalli, R., Rice, K.M., Nandyala, G., Fankenhanel, E., et al., 2015. Effect of cerium oxide nanoparticles on sepsis induced mortality and NF-kappaB signalling in cultured macrophages. Nanomedicine 10,12751288.

Sogaard, M., Andersen, J.P., Schonheyder, H.C., 2012. Searching PubMed for studies on bacteremia, bloodstream infection, septicemia, or whatever the best term is: a note of caution. Am. J. Infect. Control 40, 237-240.

Swiecki, M., McCartney, S.A., Wang, Y., Colonna, M., 2011. TLR7/9 versus TLR3/ MDA5 signalling during virus infections and diabetes. J. Leukoc. Biol. 90, 691701.

Tille, P., 2013. Bailey \& Scott's Diagnostic Microbiology. Elsevier Mosby, St. Louis.

Wu, J., Tian, L., Yu, X., Pattaradilokrat, S., Li, J., Wang, M., et al., 2014. Strain-specific innate immune signalling pathways determine malaria parasitemia dynamics and host mortality. Proc. Natl. Acad. Sci. U. S. A. 111, E511-520. 